\title{
Journal contacts
}

\section{ABOUT THE JOURNAL}

Acta IMEKO is an e-journal reporting on the contributions on the state and progress of the science and technology of measurement. The articles are mainly based on presentations presented at IMEKO workshops, symposia and congresses. The journal is published by IMEKO, the International Measurement Confederation. The ISSN, the international identifier for serials, is 2221-870X.

\section{EDITOR-IN-CHIEF}

Paolo Carbone, Italy

\section{HONORARY EDITOR-IN-CHIEF}

Paul P.L. Regtien, Netherlands

\section{EDITORIAL BOARD}

Leopoldo Angrisani, Italy

Filippo Attivissimo, Italy

Eulalia Balestieri, Italy

Eric Benoit, France

Catalin Damian, Romania

Pasquale Daponte, Italy

Luigi Ferrigno, Italy

Edoardo Fiorucci, Italy

Alistair Forbes, United Kingdom

Sabrina Grassini, Italy

Fernando Janeiro, Portugal
Konrad Jedrzejewski, Poland

Andy Knott, United Kingdom

Fabio Leccese, Italy

David Macii, Italy

Rosario Morello, Italy

Michele Norgia, Italy

Helena Geirinhas Ramos, Portugal

Pedro Ramos, Portugal

Sergio Rapuano, Italy

Dirk Röske, Germany

Alexandru Salceanu, Romania
Constantin Sarmasanu, Romania

Diego Scaccabarozzi, Italy

Lorenzo Scalise, Italy

Emiliano Schena, Italy

Enrico Silva, Italy

Marco Tarabini, Italy

Susanne Toepfer, Germany

Rainer Tutsch, Germany

Ian Veldman, South Africa

Luca De Vito, Italy

\section{FINAL EDITING}

Rosario Morello, University Mediterranea of Reggio Calabria, Italy

\section{ABOUT IMEKO}

The International Measurement Confederation, IMEKO, is an international federation of actually 39 national member organisations individually concerned with the advancement of measurement technology. Its fundamental objectives are the promotion of international interchange of scientific and technical information in the field of measurement, and the enhancement of international co-operation among scientists and engineers from research and industry.

\section{ADDRESSES}

\begin{tabular}{ll}
\hline Principal Contact & $\begin{array}{l}\text { Prof. Paolo Carbone } \\
\text { University of Perugia } \\
\text { Dept. Electr. Inform. Engineering (DIEI) } \\
\text { via G.Duranti, 93, 06125 Perugia, Italy } \\
\text { email: paolo.carbone@unipg.it }\end{array}$ \\
\hline ACTA IMEKO & $\begin{array}{l}\text { Prof. Paul P. L. Regtien } \\
\text { Measurement Science Consultancy (MSC) } \\
\text { Julia Culpstraat 66, 7558 JB Hengelo (OV), The Netherlands } \\
\text { email: paul@regtien.net }\end{array}$ \\
\hline Support Contact & $\begin{array}{l}\text { Dr. Dirk Röske } \\
\text { Physikalisch-Technische Bundesanstalt (PTB) } \\
\text { Bundesallee 100, 38116 Braunschweig, Germany } \\
\text { email: dirk.roeske@ptb.de }\end{array}$
\end{tabular}

